\title{
Sleep medicine practice adaptations
}

\author{
Marc Raphaelson, MD, FAAN, FAASM \\ Daniel B. Brown, Esq
}

\begin{abstract}
Summary
Sleep medicine has been a rapidly growing field for the last 15 years. Medicare and private payer insurers continually examine testing and treatment closely to monitor potential fraudulent practices. In this article, we explore responses by some practitioners to adapt to policy changes. In addition, we offer advice to clinicians on how to review their customary office procedures and involve patients in overcoming administrative obstacles to the diagnostic and therapeutic course agreed upon by a doctor and patient.
\end{abstract}

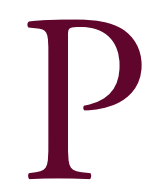

ayment policies rolled out by Medicare and commercial insurance plans often disrupt the practitioner's preferred clinical response to a patient's disordered sleep. Although clinical and scientific advances have contributed to rapid growth in sleep medicine therapy, ${ }^{1}$ the potential for fraudulent physician actions has Medicare and other insurers examining sleep testing and treatment closely. The 2013 Work Plan for the Office of the Inspector General of the US Department of Health and Human Services includes reviews of payments for polysomnography (PSG) and for continuous positive airway pressure (CPAP) replacement supplies. ${ }^{1}$

This article describes responses by some practitioners, some by one author and some by other practices, to adapt to administrative difficulties in sleep practice. We advise clinicians to consider revising their own office policies similarly.

\section{Reimbursement for sleep testing}

Medicare, state, and commercial insurer rules for sleep testing are specific, and they are subject to change without direct notification to individual physicians.

Medicare The Centers for Medicare \& Medicaid Services (CMS) governs Medicare reimbursement for sleep testing through the Medicare manuals and a national coverage determination addressing home and in-lab sleep testing. Local Medicare Administrative Contractors, or "MACs," govern the Medicare conditions for coverage of sleep testing performed in hospital labs, physician-owned labs, and stand-alone sleep labs in their jurisdictions. ${ }^{2}$ Separate federal regulations govern coverage for tests billed to Medicare by independent diagnostic testing facilities (IDTFs).

Dresevic, Gustafson, Iwrey, Kalmowitz and Pendleton, The Health Law Partners (DBB), Atlanta, GA.

Funding information and disclosures are provided at the end of the article. Full disclosure form information provided by the authors is available with the full text of this article at Neurology.org/cp.

Correspondence to: raphaels4@aol.com 


\section{When an insurer denies clinically indicated testing} or treatment, request that the patient notify the employer's human resources department and the state insurance commissioner.

Both Medicare and commercial policies often specify whether the testing center must be accredited by the American Academy of Sleep Medicine, ${ }^{3}$ the Joint Commission, ${ }^{4}$ or the Accreditation Commission for Health Care ${ }^{5}$; whether the technologist must be registered or licensed; and whether the doctor must be board-certified in sleep medicine. Important distinctions for reimbursement exist depending on the setting or type of sleep test. For example, a registered sleep technologist may or may not be required for Medicare sleep tests performed in the physician practice setting. However, a registered or licensed sleep technologist will always be required for sleep tests billed to Medicare by IDTFs.

Commercial payers Like Medicare, commercial insurance coverage rules for sleep testing are fragmented, varied, and changing rapidly. For example, some private payers require that the sleep lab be accredited as a condition to payment. More and more commercial payers are now instituting preauthorization procedures for sleep testing. These procedures favor payment for out of center testing (OOCT, also known as home, portable, or unattended sleep testing) in lieu of the more expensive in-lab PSG when clinically acceptable.

Practitioner actions To stay abreast of these changes, practitioner responses include the following:

- At least once every year, read the pertinent CMS and other insurer national and local coverage determinations (LCDs).

- Medicare and its Recovery Audit Contractors may interpret Medicare's documentation requirements more strictly than a common reading might suggest. For example, auditors could seek overpayment penalties against the sleep lab if the medical record fails to indicate the neck circumference, despite voluminous written evidence of indicators of disordered sleep. A 2013 audit in Durable Medical Equipment (DME) Jurisdiction A found overpayment liability when the sleep test data sheets were not signed, even though the physician duly signed the final interpretation letter. The practice must review documentation requirements periodically, and you should request legal representation at the start of any audit that may be problematic.

- For accredited testing centers: At least annually, check accreditation standards and revise policies and procedures for current clinical and accreditation standards.

- Monitor state requirements for technologist registration or licensure. ${ }^{6}$ In Maryland, for example, PSG technologists are required to be licensed as of October 1, 2013. In some states the technologist scope of practice may lead to a requirement that only licensed technologists perform OOCT.

- Do not perform any procedure for commercial insurance plan members until the plan issues prior authorization for the Current Procedural Terminology code of the test to be performed.

- Prepare a practice-specific prior authorization form for sleep testing to be used with commercial payers. This form should contain checklists for patient symptoms, signs, clinical diagnoses, and potential contraindications to OOCT. Most insurers should accept such a form in place of their own proprietary form. Figure 1 is an example of such a form that may be adapted for any practice.

- When clinically indicated, prior authorization requests should emphasize the need for in-lab testing. For example, OOCT generally cannot diagnose suspected periodic limb 
Figure 1 Prior authorization worksheet for sleep testing (example)

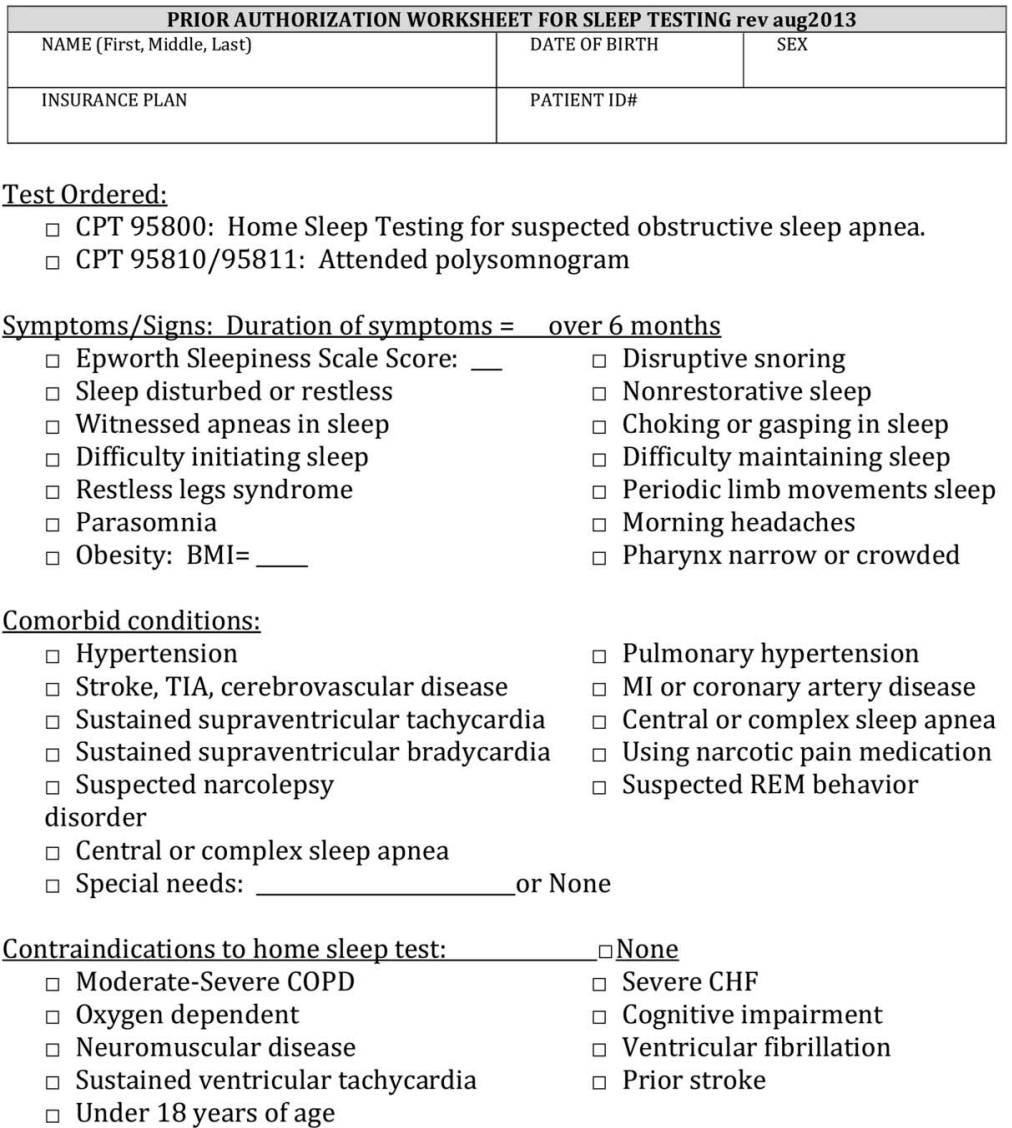

I have evaluated the patient face to face for above symptoms. This test is medically necessary.

\begin{tabular}{lr}
\hline MD Signature & MD NPI\# \\
Certified, AAN, Neurology and Sleep Medicine &
\end{tabular}

Example of a practice-specific prior authorization form for sleep testing to be used with commercial payers. This form may be adapted for any practice.

movements of sleep, and it cannot be used for multiple sleep latency testing. Any medical contraindications to OOCT should be clearly indicated.

- Request a peer-to-peer case review of any authorization denial. A denial can be reversed when the patient condition is explained in greater detail during a collegial peer-to-peer review process. Rather than participate by telephone, some practitioners simply send copies of complete office notes for the insurance reviewer. The Health Insurance Portability and Accountability Act permits covered entities such as sleep doctors and sleep labs to disclose protected health information freely for payment, treatment, and health care operations. Preauthorization fits all 3. No prior patient authorization for these disclosures is required.

- The final diagnoses from sleep testing should include clinical diagnoses. For example, a patient with insomnia, nonrefreshing sleep, and daytime sleepiness might undergo PSG that demonstrates snoring without substantial sleep apnea. The final listed diagnoses might include subjective hypersomnolence as well as primary snoring. Since some payers may not cover PSG for primary snoring, a more complete list of clinical diagnoses may help the insurer to apply coverage policy appropriately and avoid incorrectly applied denials of service. 


\section{Unlike Medicare reimbursement for sleep testing, Medicare's coverage conditions for CPAP therapy prescribed to treat obstructive sleep apnea are uniform across the country.}

- Some patients have substantial sleep apnea clinically, but their test may not support the diagnosis. For other patients a false negative test might result if there is short sleep time without REM sleep and without sleep in the supine position. In those cases repeat testing may be performed in the lab while the patient is kept in the supine position and while taking hypnotic medication if clinically indicated.

- When an insurer denies clinically indicated testing or treatment, request that the patient notify the employer's human resources department and the state insurance commissioner. This is likely to be more effective than a physician complaint. If there is a repeated pattern of incorrect service denial, have the patient speak with the payer's medical director.

\section{Reimbursement for CPAP therapy}

Medicare Medicare coverage for CPAP therapy prescribed to treat obstructive sleep apnea falls under the category of DME. DME coverage is completely separate from coverage for medical examinations and diagnostic testing, so a different set of rules apply to a patient's CPAP therapy following a medical diagnosis of sleep apnea.

Unlike Medicare reimbursement for sleep testing, Medicare's coverage conditions for CPAP therapy prescribed to treat obstructive sleep apnea are uniform across the country. Each of the 4 DME MACs has adopted the same LCD provision for Medicare CPAP coverage.

Because CMS believes that DME in general—and CPAP in particular-is subject to fraud and abuse, CPAP suppliers are subject to strict adherence to voluminous documentation requirements, most of which originate at the sleep doctor's office. Typically the DME supplier must document the physician's face-to-face visit with the patient, testing results, clinical improvement during treatment, and objective adherence to therapy. Physicians may not dispense CPAP to Medicare patients under most practical conditions.

These requirements burden both the DME supplier and the sleep physician. The supplier won't get paid unless the physician first provides the supplier with detailed notes of his or her initial physical examination, along with the results of the sleep test and evidence that the physician interpreting the test meets certain requirements. Then the supplier must obtain a record of a follow-up face-to-face examination after CPAP therapy begins to document that the therapy is effective and that the patient is regularly using the CPAP equipment. If the physician fails to deliver complete documents or perform the required examinations and services, the CPAP supplier will not get paid and the patient may not get needed therapeutic equipment.

Commerical insurance CPAP coverage from commercial insurance companies varies from company to company. Many payers follow most, if not all, of the Medicare requirements for CPAP coverage. Many insurers will pay for CPAP coverage if the respiratory disturbance index is $5 /$ hour or higher, whereas Medicare will pay for CPAP coverage only if the apnea/hypopnea index is 5/hour or higher. Physicians may dispense CPAP to some privately insured patients, but the practice is regulated by payer contracts and both federal and state fraud rules.

\section{Practitioner actions}

To stay current with these changes, practitioner responses include the following:

- Prepare your own practice-specific standard CPAP order form that combines prescription and certificate of medical necessity, rather than using a separate proprietary form for 
Figure 2 Positive airway pressure (PAP) prescription and certificate of medical necessity (example)

PAP PRescription AND Certificate of Medical NeCEssity rev aug2013

Patient name:

DOB:

DO NOT DISPENSE PAP UNLESS YOU WILL PROVIDE DATA

At day 31-35 - and prn - print and deliver to patient \& me, adherence data including: AHI \& Percent nights used 4 h or more \& $90 \%$ IPAP \& Leak data

RX/CONTINUE DEVICE BELOW: WITH E0562, HEATED HUMIDIFIER

SET Ramp 4 cwp over 20 min. Raise minimum ramp pressure prn.

$\begin{array}{ll}\square \text { Auto-adjusting CPAP E0601: } & \text { Low limit__upper limit } \\ \square \text { Auto-adjusting Bilevel PAP E0470: } & \text { Min EPAP_ Max IPAP }\end{array}$

$\checkmark$ Servoventilator Bilevel PAP E0471: Default rate.

Min EPAP Max IPAP PS min PS Max

$\square$ Show patient how to set minimum/maximium pressure for comfort

RX: $\square$ INCLUDE OR $\square$ REPLACE PRN FOR 12 MONTHS:

\begin{tabular}{lll|lll} 
A7027 & Oro/Nasal Mask & 1per3Mo & A7036 & Chinstrap & 1per6Mo \\
A7028 & Oral Cush Repl & 2perMo & A7037 & Tubing for PAP & 1per3Mo \\
A7029 & Nasal Pillows Repl & 2perMo & A7038 & Filter Disposable & 2perMo \\
A7030 & Full Face Mask & 1per3Mo & A7039 & Filter Nondisposable & 1per6Mo \\
A7031 & FF Mask Cush Repl & 1perMo & A7044 & Oral Interface & 1per3Mo \\
A7032 & Nasal Cush Repl & 2perMo & A7045 & Exhalation Port Repl & NA \\
A7033 & Nasal Pillows Repl & 2perMo & A7046 & Water Chamber & 1per6Mo \\
A7034 & Nasal Interface Mask & 1per3Mo & A4604 & Tubing w Integ Heat & 1per3Mo \\
A7035 & Headgear & 1per6Mo & & & \\
Mask: & \multicolumn{3}{c}{ Size S M L. Fit \& RX alternate mask or nasal pillows prn. }
\end{tabular}

DIAGNOSIS:

$\square 327.23$ Obstructive sleep apnea $\mathrm{AHI}=$

$\mathrm{RDI}=$

327.21 Central sleep apnea

$\square$ CPAP tried \& proven ineffective based on a therapeutic trial

$\square$ AHI 5-14 with daytime hypersomnolence, impaired cognition, mood disorder, insomnia, hx stroke, hypertension, or ischemic heart disease

ATTESTATIONS:

I attest: I have visited face-to-face with this patient and evaluated signs \& symptoms.

al attest: The patient has had a polysomnogram or home sleep test that:

1. Meets Medicare criteria for sleep apnea diagnosis and CPAP coverage.

2. Was performed according to Medicare standards by an entity that qualifies as a Medicare provider of sleep tests and is in compliance with all applicable state regulatory requirements.

Was evaluated with Medicare-specified rules for scoring apneas/hypopneas and reporting AHI and RDI.

4. Was interpreted by a physician who meets Medicare qualifications.

4. Whis interpreted by a physician who meets is medically necessary for this patient's well-being. It is reasonable and necessary in reference to accepted standards of medical practice for this condition, and it is not prescribed as a convenience. Estimated period of medically necessity: 99 months or lifetime.

- I have visited face-to-face with this patient between the 31st and 91st day after PAP therapy was initiated.

a Symptoms of sleep apnea have improved.

$\square$ Objective adherence during 30 days for 4 hours or more is $\%$.

$\square$ Replacement device. I attest: The patient is currently on PAP and is adherent for therapy.

MD Signature MD NPI\#__ Therapy Start Date/Signature Date

Certified, AAN, Neurology and Sleep Medicine

Example of a practice-specific standard PAP order form that combines prescription and certificate of medical necessity information. This form may be adapted for any practice.

each provider. Medicare does not accept "blanket orders" or minimum check-the-box order forms prepared by DME suppliers. The form and language should meet CMS standards, including the appropriate Healthcare Common Procedure Coding System codes. If a supplier cannot dispense DME based on your practice's form, ask the supplier to specify the deficiency in your form. If there is none, then the supplier should accept the form or refer the patient to another supplier. Figure 2 is an example of such a form that may be adapted for any practice.

- Design your electronic health record to include CPAP supply prescriptions to be sent to suppliers.

- Because the prior authorization process may delay treatment, start the clock by ordering DME immediately when interpreting results of diagnostic or titration studies. Most practices require a follow-up clinical visit to review diagnostic findings and to introduce CPAP desensitization, so the DME form can include an order not to dispense therapy 
until the patient has completed the return visit. That allows the DME supplier to start the prior authorization process, but the patient does not start therapy until the practitioner and patient have finalized their therapeutic decision.

- While some physicians will download and print adherence and efficacy information from a CPAP memory chip or read data directly from the device, most will not. You may require the DME supplier to print out data on demand and deliver it to the patient for review with you at a subsequent visit. Technologically savvy patients may print their own data using single-user software from the equipment manufacturer or SleepyHead, ${ }^{7}$ described online as a free open-source program.

- Most practices now schedule 2-3 follow-up visits to address patient concerns and promote adherence during the Medicare 90-day trial window.

- Some practices schedule CPAP follow-up visits during a single half day and contract with a supplier to attend the clinic, under conditions that meet anti-kickback laws. When practicable, this arrangement allows the supplier to deliver efficacy and adherence data and to dispense new equipment in conjunction with office visits.

- As CPAP payments have come down, some DME companies may become less responsive to patient needs. Frustrated patients are increasingly looking to the sleep medicine practice for services formerly provided by the DME company. Utilize nurse practitioners and physician assistants to maximize practice efficiency and attention to patients. Consider "incident-to service" billing for technologists to provide CPAP initiation and management, desensitization, mask fittings, and other services when other billing criteria are met. (Medicare has proposed changes to these rules in the proposed physician fee schedule for $2014 .{ }^{8}$ ) Practice staff may perform a "PAP-NAP" for CPAP desensitization and initiation when clinically warranted.

- Consider whether your local payer and legal environment favors opening your own DME company.

\section{Oral appliance therapy}

Oral appliance therapy (OAT) for sleep apnea is also DME. List prices are high, and insurance coverage is less universal than for CPAP.

Practice responses include the following:

- Counsel patients to find a board-certified dentist, or one with special interest in sleep apnea, through the Web site for the American Academy of Dental Sleep Medicine.

- Counsel patients to check prices and possible participation in medical—not dentalinsurance plans for available expert dentists. Counsel patients to check OAT coverage separately with their insurer before committing to a course of treatment.

- Where permitted by state corporate practice doctrines and the legal scope of practice of physicians and dentists, some sleep medicine clinics dispense OAT, while some recruit a dentist to practice on site.

\section{Sleep medications}

Medications for sleep disorders are expensive. Increasingly insurers require prior authorization for stimulant and hypnotic medications. Often the insurer requires only the clinical diagnosis to authorize a prescription. For some insurers the office personnel may spend more than an hour negotiating telephone menus to get the needed forms.

Practice responses include the following:

- Require the patient to provide forms from his or her own insurer for prior authorization, and then the practice can complete them promptly. The insurer should send forms directly to the patient, and then the patient should send them to the practice. This allows the patient to supervise the entire process and to recognize the source of any delay, whether at the insurer or at the practice. 
- Rather than complete a separate form, some practitioners send copies of complete office notes for review by the insurance viewer. The practice also may provide a copy of the note to each patient so he or she may submit it for medication authorization review.

- Some practices require repeat office visits for each refill of a Schedule II medication, and some offices require that those refills be picked up at the office. Other practices will mail refills to the pharmacy or to the patient.

People with sleep disorders are subject to federal and state regulations on driving, piloting, and operating heavy equipment. They may need specific evaluation for work fitness or limitations or for necessary work or school accommodations.

Practice responses include the following:

- Most practices require a written list of duties and physical requirements for any job-related inquiry and a written list of questions to be addressed for a specific work circumstance.

- Some practices require that administrative forms be completed during a face-to-face visit. This allows patient input into medical recommendations that may be nuanced or contentious. Forms for work release or limitation, for example, may be completed most accurately during discussion between the patient and physician.

A well-documented health record is increasingly necessary as Medicare Advantage and other insurers may perform annual chart audits. Each follow-up note must include sufficient information to justify the billing codes and usually should include a complete problem list. The record is increasingly valuable to streamline prior authorizations for testing and treatment, particularly if it includes the following:

- Up-to-date documentation of all prior sleep testing

- All medications and treatments that may not have been tolerated, effective, or accepted

- Serial treatment adherence and efficacy data

- Chronologic listing of sleepiness scores and vital signs

The old saw that reimbursement drives health care in America has particular application to sleep medicine. Practitioners can't make payment policy, but they can work with their patients to improve access to needed care under difficult circumstances.

\section{REFERENCES}

1. 2013 Work Plan, Office of Inspector General of the US Department of Health and Human Services. Available at: http://oig.hhs.gov/reports-and-publications/archives/workplan/2013/Work-Plan-2013.pdf. Accessed September 7, 2013.

2. Medicare coverage database for National Coverage Determinations and Local Coverage Determinations. Available at: http://www.cms.hhs.gov/mcd/overview.asp. Accessed September 7, 2013.

3. American Academy of Sleep Medicine Center Accreditation. Available at: http://www.aasmnet.org/ accreditation.aspx. Accessed September 7, 2013.

4. The Joint Commission: Seeking Sleep Center Accreditation. Available at: http://www.jointcommission. org/accreditation/ahc_seeking_sleep_centers.aspx. Accessed September 7, 2013.

5. Accreditation Commission for Health Care: Sleep Lab Program Accreditation Process. Available at: http://www.achc.org/programs/sleep-lab/sleep-lab-accreditation-process. Accessed September 7, 2013.

6. American Association of Sleep Technologists State Legislative Updates. Available at: http://www.aastweb. org/articles.aspx?id=3353. Accessed September 7, 2013.

7. SleepyHead software. Available at: http://sourceforge.net/apps/mediawiki/sleepyhead/index.php?title=Main_ Page. Accessed September 7, 2013.

8. Medicare Proposed Rule, Physician Fee Schedule 2014. Available at: http://www.cms.gov/Medicare/ Medicare-Fee-for-Service-Payment/PhysicianFeeSched/PFS-Federal-Regulation-Notices-Items/CMS-1600-P. html. Accessed September 7, 2013.

\section{ACKNOWLEDGMENT}

The authors thank Amy J. Aronsky, DO, FCCP, FAASM, CBSM, and Mark Miller, MD, FAASM for critical review of the manuscript. 


\section{STUDY FUNDING}

No targeted funding reported.

\section{DISCLOSURES}

M. Raphaelson serves on the Scientific Advisory Board and speakers' bureau and has received funding for travel and speaker honoraria from Jazz Pharmaceutical; practices sleep medicine and bills for related services; is president of Marc Raphaelson, MD, PA, Inc., which collects fees from governmental and commercial insurers; serves as Medical Director for SleepMed, Inc., a for-profit entity providing sleep medicine testing and treatment; and has prepared affidavits for Sleep Information Systems, a nowdissolved for- profit entity. D. Brown is a practicing attorney representing clients including physicians and sleep laboratories and bills for related services; has received speaker honoraria from Fisher \& Paykel Healthcare and the Atlanta School of Sleep Medicine and Technology; and serves as a consultant for Sleeping Well and LLC Fisher \& Paykel Healthcare, Inc. Full disclosure form information provided by the authors is available with the full text of this article at Neurology.org/cp.

\section{Related articles from other AAN physician and patient resources}

Neurology ${ }^{\circledR} \quad$ www.neurology.org

The coming crisis: Obtaining care for the growing burden of neurodegenerative conditions May 21, 2013;80:1989-1996.

\section{Continuum ${ }^{\circledR} \quad$ - www.ContinuumJournal.com}

Coding for sleep disorders

February 2013;229-235.

$$
\text { Neurology Today }{ }^{\circledR}
$$

- www.neurotodayonline.com

In Practice: More Death By A Thousand Cuts: Neurology in the Crosshairs as CMS Seeks to Slash Reimbursement for "Bread and Butter" Codes March 15, 2012;12:13-16. 